\title{
NOUVELLES RECHERCHES SUR LA MORPHOLOGIE ET SUR L'ÉVOLUTION D'ICOSIELLA NEGLECTA (DIESING 1851), FILAIRE COMMUNE DE LA GRENOUILLE VERTE
}

\author{
Par C. DESPORTES
}

La connaissance de l'évolution de filaires parasites de Batraciens est une acquisition récente. En effet, les cycles évolutifs établis - au moins expérimentalement - pour de telles filaires apparaissent pour la première fois dans la littérature en $\mathrm{r} 939$, à la suite des travaux publiés par 0 . R. Causey : ces cycles sont ceux de trois espèces appartenant à un même genre, Foleyella Seurat, et parasites de grenouilles américaines.

Pour l'une des trois espèces citées par Causey, Foleyella dolichoptera $\mathrm{E}$. E. Wehr et $\mathrm{O}$. R. Causey 1939 , on peut noter que la découverte des stades larvaires chez les hôtes vecteurs - des Culicidés a précédé sinon la découverte, au moins la description de la forme adulte.

Il en a été tout autrement d'Icosiella neglecta (Diesing I 85 I), filaire banale de la Grenouille verte, dont les premiers spécimens semblent déjà figurer dans le Catalogue des Entozoaires du Musée de Vienne de I8I I, dont la microfilaire fut découverte en I84I, mais sur l'évolution de laquelle toutes les hypothèses émises et recherches entreprises depuis lors sont demeurées stériles.

C'est pourquoi nous avons cru intéressant de donner dans le présent travail les quelques résultats obtenus à la suite d'une première série de recherches sur le cycle d'Icosiella neglecta (Diesing), entreprises à la Station de Parasitologie expérimentale de Richelieu (Indreet-Loire).

Nous voulons cependant auparavant rappeler brièvement l'historique de la question et apporter quelques précisions sur la morphologie de ce nématode, puisqu'il y a peu d'années seulement, le genre auquel il appartient a été mis en doute et que les synonymies d'I. neglecta ont été l'objet de plusieurs confusions.

Annales de Parasitologie, T. XVIII, Nos $1-2-3$. $-194 \mathrm{I}, \mathrm{p}$. 46-67. 


\section{Historique}

Enumérant la succession chronologique des travaux concernant cette filaire, nous la voyons figurer pour la première fois dans le Catalogue des Entozoaires du Musée de Vienne de 18 I I : les spécimens, récoltés sous la peau de Rana esculenta, dix-neuf fois en été, une fois en hiver, à la dissection d'un total de r.29o grenouilles, sont étiquetés "Filaria sp. d. pell. "; un des exemplaires est envoyé au Muséum de Paris.

Cependant, en ${ }_{1} 8$ I $9_{9}$, Rudolphi décrit sous le nom de Filaria rubella Rud., un nématode rougeâtre trouvé d'abord par Klug, à Berlin, dans l'estomac de Rana temporaria, puis, par Rudolphi lui-même, enkysté dans le mésentère et dans de petits tubercules de la paroi externe de l'estomac et de l'intestin de la Grenouille verte : Rudolphi émet alors l'hypothèse que Filaria ranæ esculentæ du Catalogue des Entozoaires de Vienne, espèce non étudiée par lui, et qu'il range parmi les espèces douteuses, pourrait être la même espèce que Filaria rubella.

Puis Valentin, dans un travail publié en I $84 \mathrm{I}$, signale l'existence, dans le sang de la patte de la Grenouille commune, d'Anguillula intestinalis : l'auteur considère la présence de ces vers dans le sysṫ̀me circulatoire comme étant liée aux migrations, de faible durée, des formes jeunes se dirigeant vers les organes propres à leur développement.

A peu près à la mème époque, Vogt (1842) rencontre les mêmes petits vers dans les vaisseaux des grenouilles, mais signale en outre leur présence dans des kystes viscéraux et péritonéaux; enfin, Vogt découvre, entre le foie et le péricarde, deux vers adultes de plus de 27 millimètres; il en conclut à une évolution comportant trois stades : le premier dans les vaisseaux; puis un stade d'accroissement dans les kystes; enfin libération des formes adultes dans la cavité abdominale où s'effectue la reproduction.

Une étude résumée des travaux précédents se trouve dans l'Hisloire des Helminthes de F. Dujardin (1845). L'hypothèse de Rudolphi est alors acceptée par ce dernier auteur et c'est sous le nom de "Fil. rougeâtre. Fil. rubella Rud. " que figurent, en même temps que les exemplaires de Rudolphi, d'une part la filaire envoyée en $18 \mathrm{r} 6$ par le Musée de Vienne à Paris (étudiée et décrite par Dujardin), d'autre part les petits nématoïdes du sang découverts par Valentin puis par Vogt.

Cependant C. M. Diesing n'acceptera pas cette synonymie : dans 
Systema Helminthum, de $\mathrm{r} 85 \mathrm{r}$, une distinction est établie entre la filaire sous-cutanée de la Grenouille verte, Filaria ranæ esculentæ Cat. Ent. Vienn. 24, que Diesing nomme Filaria neglecta Diesing, et la filaire enkystée dans le mésentère et dans les parois de l'estomac et de l'intestin, Filaria rubella Rudolphi, de laquelle Filaria ranæ esculentæ Valentin, espèce douteuse, serait peut-être un stade évolutif: "An Filaria rubella ... in evolutione versans?... ".

Le rapport entre les formes du sang et les vers adultes apparaîtra beaucoup mieux, trois ans plus tard, à la suite d'une constatation de Vulpian (1854). Cet auteur remarque en effet que les grenouilles renfermant les " nématoïdes " du sang hébergent simultanément, au milieu des gros vaisseaux qui se dirigent du cœur vers le bras et des nerfs du plexus brachial, plus rarement dans les muscles sous-hyoïdiens, des vers femelles renfermant des embryons semblables à ceux du sang. En outre, il ne retrouve pas, malgré des recherches attentives, les formes enkystées signalées par Vogt. Ces très importantes constatations de Vulpian ne sont malheureusement pas accompagnées de description de l'adulte et l'étude bibliographique n'a pas été approfondie, de sorte que rien, sinon la localisation des adultes dans l'hôte, ne permet de penser qu'il s'agit de Filaria neglecta Diesing plutôt que d'une autre espèce; il semble, au contraire, à la lecture de ce travail, que Vogt et Vulpian soient les, premiers auteurs qui aient signalé des formes adultes chez les grenouilles.

C'est pourquoi, s'en référant à la publication de Vulpian, de Nabias et Sabrazès $\left(189^{2}\right)$ ont écrit que la découverte de l'embryon de " la Filaire du sang des grenouilles " a précédé celle de l'adulte. Le travail de Nabias et Sabrazès est pourtant extrêmement important : outre la découverte du mâle et une bonne description des adultes, ces helminthologistes précisent la localisation chez l'hôte de la filaire : "le véritable habitat de ces animalicules est le tissu conjonctif sous-cutané et intramusculaire ". Il est donc bien exact que la filaire dont parlent ces auteurs est la Filaria neglecta de Diesing. Enfin la notion, dans l'évolution de la filaire, d'hôte intermédiaire " qu'il faudra chercher parmi les animaux qui vivent dans les mares aux grenouilles " apparaît pour la première fois dans ce mémoire.

Pourtant, en 1895 , dans l'ouvrage intitulé Les Vers du Sang de R. Blanchard nous trouvons de nouveau réunies - " par erreur évidemment ", a dit Railliet (1916) - les espèces décrites par Rudolphi, Valentin, Vogt, Vulpian et de Nabias et Sabrazès sous le même nom de "Filaria rubella Rudolphi, 1819 , syn. : Filaria ranæ esculentr, Valentin, i84r ").

La même erreur est commise en $19 \circ 9$ par 0 . Linstow, qui désigne 
sous le nom de Filaria rubella Rud. la filaire qui habite " im Unterhautzellgewebe und intramuskulären Bindegewebe von Rana temporaria L. und Rana esculenta L. " et, en 1912 , par A. Porta, dans une note concernant des recherches sur la transmission par des Hirudinés de Filaria rubella Rud., ce dernier nom étant appliqué à des nématodes de la mème espèce que ceux étudiés par de Nabias et Sabrazès, c'est-à-dire les vrais neglecta.

Mais, dans une étude sur les filaires des Batraciens, de I916, A. Railliet, comme Diesing en $\mathrm{r} 85 \mathrm{I}$, sépare Filaria neglecta Dies. (F. ranæ esculenlæ C. E. V.), filaire commune du tissu conjonctif des grenouilles, de Filaria (?) rubella Rud. 1819, dont le type habite l'estomac de Rana temporaria.

La mème année, Filaria rubella Rudolphi e. p. est mise par L. G. Seurat (rgr6) en synonymie douteuse de Filaria jubæ Seurat. La synonymie est confirmée l'année suivante (Seurat 1917). Cet helminthologiste définira en outre le genre Icosiella, dont I. neglecta (Diesing I85I) est le type. L'étude morphologique faite par Seurat semble parfaitement justifier la création du nouveau genre, mais nous regrettons de ne pas voir la description accompagnée de figures.

Une reproduction de cette diagnose du genre Icosiella, donnée par G. Seurat, se trouve dans Nematode Parasites of Verlebrates (Yorke et Maplestone r926) et dans The Nematoda as Parasites of Amphibia de A. C. Walton (1935) (r).

Cependant E. E. Wehr ( 1935 ) considère (peut-être par suite du manque de figures détaillées de ce ver) le genre auquel il appartient comme incertain : on voit en effet mentionné, parmi les Dipetalonematinæ, "? Icosiella, $\operatorname{Ig}^{1} 7$ " (2).

Il devenait donc nécessaire pour nous, avant de poursuivre des recherches sur l'évolution de la filaire de Rana esculenta, d'entreprendre une détermination précise des exemplaires récoltés. Nous avons pu ainsi nous convaincre que l'ensemble des caractères de ces vers était propre à l'espèce $I$. neglecta telle qu'elle a été définie par Seurat.

En particulier, rien ne la rapproche des espèces parasites de Batra-

(1) Walton met simultanément Filaria rubella Rud. en synonymie d'Icosiella neglecta (Dies. 1851) et de Filaria juba Seurat 1916. Il rapporte également à la première espèce des microfilaires voisines de "Filaria Ranae esculentae "Valentin, décrites par Mathis et Léger (1911).

(2) E. E. Wehr (p. 86) a remplacé la sous-famille des Setarinae Yorke et Maplestone 1926, par celle des Dicheilonematinae E. E. Wehr, dont il supprime les genres Dipetalonema et Icosiella " so different from Serratospiculum and Dicheilonema that they cannot possibly be placed together... " Pourtant I. Skrjabin et N. P. Schikhobalova (r936) adoptent encore la classification de Yorke et Maplestone pour ces deux genres.

Annales de Panastrologie, T. XVIII, Nos $1-2 \cdot 3 .-194 \mathrm{I}$. 
ciens appartenant aux genres Foleyella et Chandlerella, dont les femelles sont amphidelphes et dont les mâles présentent soit des papilles génitales bien visibles, souvent accompagnées d'ailes caudales (Foleyella), soit des spicules égaux (Chandlerella).

Il est, par contre, assez difficile de différencier morphologiquement cette filaire d'une espèce américaine, décrite par A. C. Walton (1927), Icosiella quadrituberculata (Leidy 1856 ) [ = Icosiella solitaria (Leidy 1856)], parasite de Rana catesbeiana et de Rana pipiens (I).

Néanmoins nous considérons la localisation de cette grande espèce dans des kystes mésentériques comme un argument suffisant pour l'écarter de notre diagnose et déterminons les spécimens récoltés comme étant typiquement Icosiella neglecta Seurat 1917 .

\section{Morphologie.}

Afin d'éviter une description qui ne serait qu'une simple répétition de celle donnée par G. Seurat, nous nous bornerons à donner de ces vers des figures exécutées à la chambre claire.

Dans le détail cependant, il y a lieu de préciser les quelques points suivants :

I $^{\circ}$ Nous avons noté, qu'en général, la longueur des femelles était supérieure à 20,950 , chiffre publié par Seurat, et plus conforme aux longueurs de 25 à 3 I mm. enregistrées par de Nabias et Sabrazès : nos plus grands exemplaires atteignent en effet 37,5 et $39 \mathrm{~mm}$; une femelle de $24 \mathrm{~mm}$. renferme des œufs et des embryons; la plus petite que nous possédions, longue de $19 \mathrm{~mm}$., renferme seulement des œufs en voie d'évolution. La longueur des mâles que nous avons récoltés oscille entre ro et I $\mathrm{mm}$., cependant un spécimen atteint I $3 \mathrm{~mm}$. 5 .

$2^{\circ}$ L'observation sur le vivant (exceptionnellement sur des exemplaires fixés) de l'extrémité antérieure de ces vers montre, accompagnant les papilles submédianes et les quatre dents, l'existence de petites saillies situées latéralement, une de chaque côté entre les papilles submédianes dorsale et ventrale, très antérieurement : il se peut que ces faibles organes représentent les amphides, extrêmement réduites chez ces filaires (fig. $2 \mathrm{~A}$ et $2 \mathrm{~B}$ ).

$3^{\circ}$ Nous n'avons jamais observé sur nos exemplaires de réceptacles séminaux ovoïdes, ainsi que les a définis Seurat, mais cylindriques et se continuant graduellement par les utérus (fig. I B).

(I) La synonymie de $I$. quadrituberculata et de $I$. solitaria, appelées primitivement Isociella par Walton, est donnée par cet auteur en 1935 . 


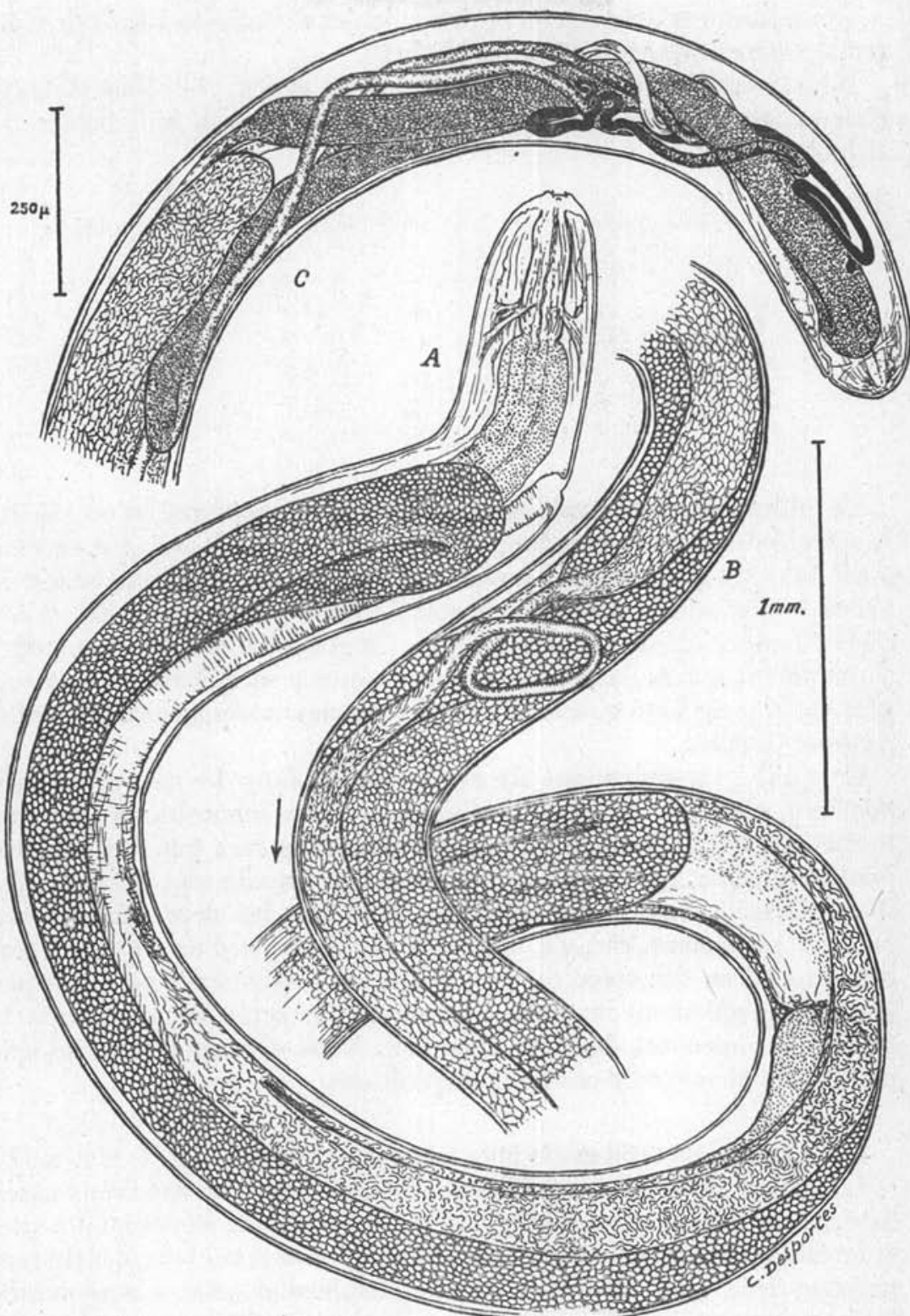

Fıg. 1. - Icosiella neglecta (Dies. 185i ). - A, extrémité antérieure d'une femelle de $29 \mathrm{~mm}$.; B, corps de la même femelle au niveau d'un des oviductes : ce tube se prolonge graduellement par le réceptacle séminal cylindrique qui se continue lui-même antérieurement (en direction de la flèche) par l'utérus; $\mathrm{C}$, extrémité postérieure d'une jeune femelle de $24 \mathrm{~mm}$. 
$4^{\circ}$ La forme et les caractères des spicules d'Icosiella neglecta méritent également une attention particulière.

Nous avons étudié ces spicules chez sept mâles : les dimensions de chacun des spicules chez six d'entre eux étaient, en millimètres, les suivantes :

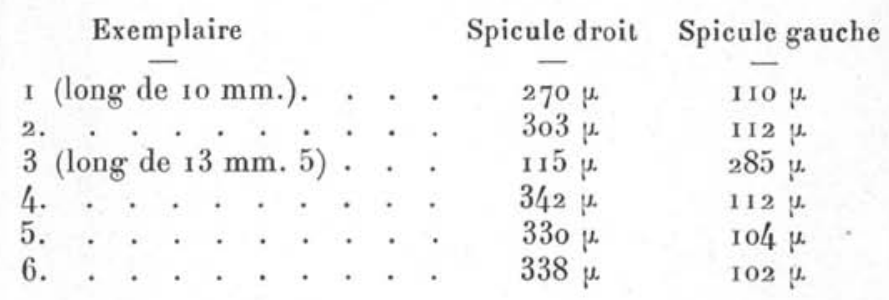

Ce tableau permet immédiatement de se rendre compte qu'en général le spicule droit est le plus long (voir aussi fig. 2-D et 2-E) : il en était ainsi dans six cas sur sept examinés, mais dans le dernier cas $\left(\operatorname{cas} \mathbf{n}^{\circ} 3\right.$ ) existe la disposition inverse : le spicule droit est le plus petit. Alors que cette dernière disposition est presque générale chez les Nématodes à spicules inégaux, la première, dans laquelle le spicule droit est le plus long, a déjà été observée : elle existe notamment chez Helerakis gallinx Gmelin.

Ce qu'il y a cependant de remarquable dans le cas d'Icosiella neglecta, c'est de voir simultanément les deux dispositions chez une même espèce : la seconde est-elle vraiment plus rare que la première, peut-être mème tératologique? ne disposant que de sept exemplaires, il est difficile d'avoir une opinion. Toutefois les deux dispositions existent réellement, car, s'il s'agissait par exemple d'un simple déplacement de l'un des spicules, l'asymétrie du petit spicule ne serait pas elle-même également inversée, comme on l'observe en comparant le spicule le plus court dans les deux cas. Nous décrirons donc les spicules dans chacune de ces deux dispositions.

I. - Le spicule droit est le plus long (cas observé six fois) (fig. 2-D).

Le spicule droit est essentiellement constitué par un axe creux assez lisse, le capitulum ne se distinguant pas du calamus, légèrement arqué et terminé distalement par un long biseau (lamına) sur les côtés duquel naissent deux ailes, droite et gauche, faiblement striées, soutendues le long de leur bord libre par une côte longitudinale très grêle. Ces deux ailes dépassent longuement la pointe du biseau et se soudent alors l'une à l'autre le long de leur bord dorsal, bord qui prolonge la 

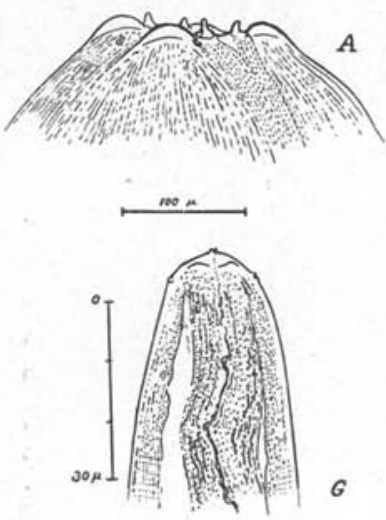

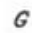

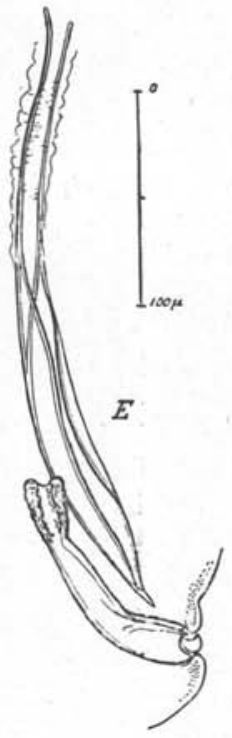

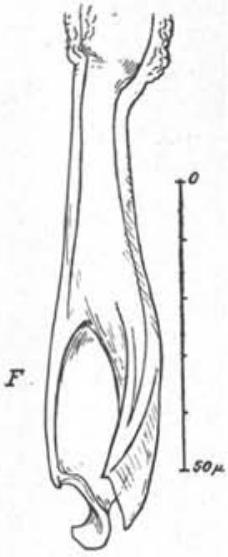
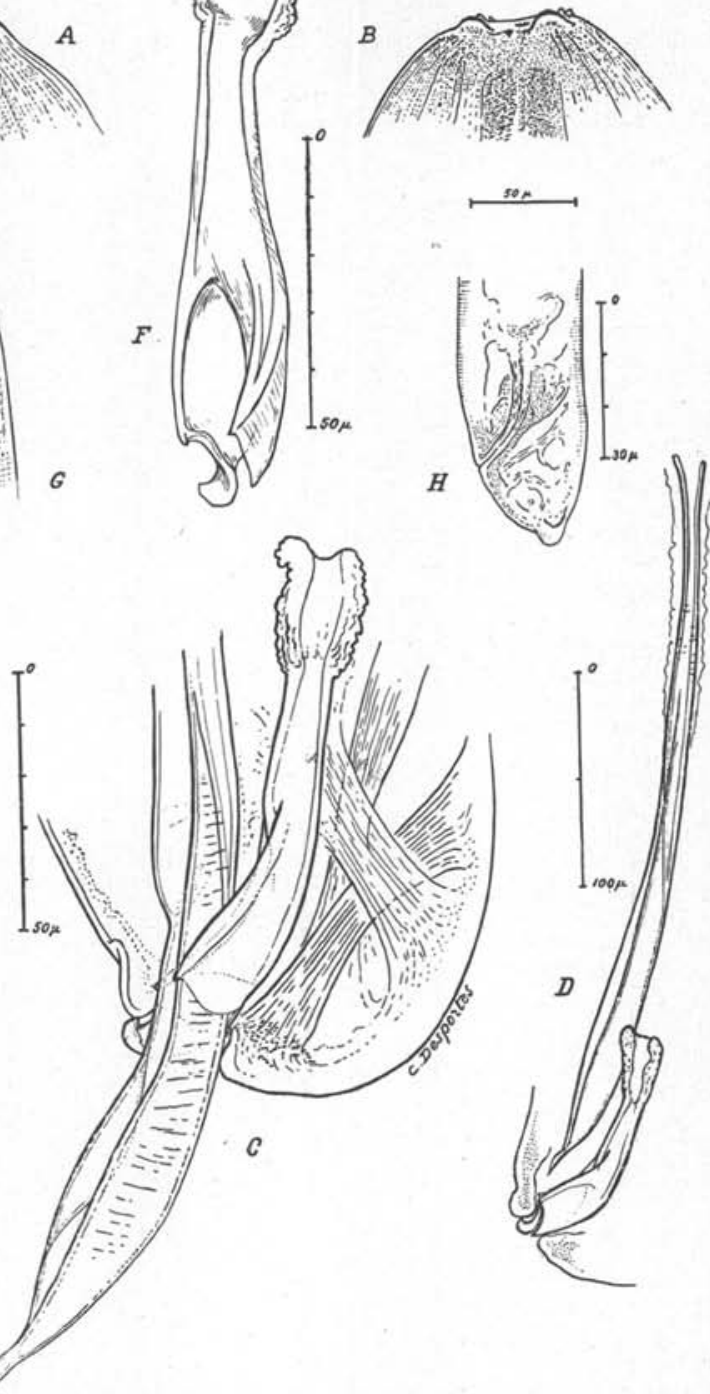

Fig. 2. - A-F, Icosiella neglecta (Dies. i 851). - A, vue latéro-dorsale de la calotte céphalique de la femelle; $B$, vue latérale de la calotte céphalique du mâle; C, extrémité postérieure du mâle : le grand spicule est engagé dans la gouttière formée par les ailes du petit spicule; D, disposition spiculaire rencontrée dans six cas examinés : le grand spicule est à droite; E, disposition spiculaire rencontrée dans un cas : le grand spicule est à gauche : l'inversion dans la disposition des spicules est accompagnée d'une inversion dans la forme du petit spicule; F, petit spicule vu de face; G, extrémité antérieure d'une des larves infectieuses spontanément hébergées par Forcipomyia velox; H, extrémité postérieure de la même larve. 
pointe du biseau, pour constituer une gouttière profonde ouverte ventralement et terminée par une fine languette.

Le spicule gauche comprend également un axe creux et deux ailes, mais l'axe est à peu près rectiligne, granuleux et épaissi dans son tiers proximal (capitulum); il se termine distalement par un court biseau.

De la base du biseau partent deux côtes arquées, épaisses, franchement obliques par rapport à l'axe du spicule, soutendant des ailes larges dépassant faiblement la pointe du biseau et non soudées l'une à l'autre par leur bord dorsal au delà de cette pointe. L'aile gauche (nous appelons aile gauche celle qui se trouve à gauche dans le corps du ver) de ce spicule, la plus courte, a la forme d'une lame subtriangulaire transparente et porte, à l'extrémité de la côte qui la soutend, un très petit bec; l'aile droite, également large, mais plus longue, présente deux becs : le premier, très faible, termine la côte qui soutend cette aile et est symétrique de celui de l'aile gauche; le second, postérieur, bien arqué, semble constitué par la partie distale libre, épaissie et repliée en crochet de l'aile droite (fig. 2-F).

II. - Le spicule gauche est le plus long (cas unique) (fig. 2-E). - Nous n'observons aucune différence entre ce cas et le précédent en ce qui concerne la morphologie du grand spicule, les deux moitiés latérales de ce spicule étant à peu près symétriques, mais nous notons par contre, pour le petit spicule, que l'aile qui porte le grand crochet terminal n'est plus la droite mais la gauche. En d'autres termes, dans chacun de ces deux cas, le grand crochet du petit spicule est toujours situé du même côté que le grand spicule.

Dans quatre des sept cas examinés, où le grand spicule n'était pas profondément retiré, il passait dans la gouttière du petit spicule, dont il émergeait distalement en passant par la fente ménagée entre les deux ailes : les gouttières des deux spicules étaient alors orientées dans le même sens - c'est-à-dire ouvertes ventralement - et emboîtées l'une dans l'autre de telle manière que les còtes bordant les ailes du grand spicule semblaient glisser sous les petits becs terminant les côtes du petit spicule (fig. $2-\mathrm{C}$ ) : en somme tout se passe morphologiquement comme si, en l'absence de gorgeret, le petit spicule constitue le guide du grand spicule ( $\mathrm{I}$ ). L'examen sur le vivant permettra seul d'avoir une certitude sur ce point.

Toutefois le petit spicule lui-même n'occupe pas une position fixe :

(I) Nous savons qu'il existe, par contre, des Nématodes à spicules inégaux chez lesquels le grand spicule abrite dans sa gouttière la pointe du spicule le plus court : nous signalons cette disposition chez Hastospiculum onchocercum (Ces Annales, sous presse). 
il s'évagine et sur trois des quatre spécimens précédents (en alcool à $70^{\circ}$ ), le large bec de ce spicule était appliqué sur la lèvre épaisse qui borde antérieurement le cloaque. Peut-ètre, lors de l'accouplement, ce large crochet pénètre-t-il dans la vulve de la femelle et maintient-il cet orifice en contact avec le cloaque, agissant à la manière d'un crampon - " a large hook which would be well adapted as a holdfast " ont écrit B. G. et M. G. Chitwood ( rg38) parlant de ce crochet chez les Nématodes à spicules inégaux - ?

N'ayant jamais rencontré de couples d'Icosielles, il ne nous a pas été permis de vérifier dans le cas particulier une telle hypothèse, néanmoins on peut remarquer que, si elle est exacte, le rôle joué par le petit spicule vis-à-vis du grand prend une importance considérable.

\section{Premières recherches sur la transmission par Forcipomyia velox Winn. d'Icosiella neglecta (Diesing).}

Dès 19o6, notre maître, le professeur E. Brumpt (communication verbale), avait remarqué, au cours d'expériences sur la transmission par Helobdella algira des trypanosomes (Tripanosoma inopinatum) de grenouilles, que les embryons d'lcosielles ne tardaient pas à succomber chez cette sangsue; il constatait les mèmes faits chez Hemiclepsis marginata, Hiemopis sanguisuga et Hirudo officinalis. Les déductions de A. Porta (1912) étaient donc inadmissibles (I).

Ces résultats négatifs orientèrent les recherches vers d'autres vecteurs, en particulier vers les Culicidés (2). Si beaucoup d'expériences ont été faites dans ce sens par différents parasitologues, aucun travail ne semble avoir été publié et rien ne permet de conclure à l'heure actuelle à la transmission possible d'Icosiella neglecta par quelque culicidé. Mais, nous ne rejetons pas non plus d'une manière absolue cette transmission, puisque la non-transmission n'a pas davantage été démontrée. Les difficultés auxquelles on se heurte en essayant de faire piquer les grenouilles par les Culicidés n'existent certainement pas toujours dans la nature, il suffit de se rappeler le cycle des différentes Foleyella de Batraciens par Culex et Aedes pour s'en rendre

(1) A. Porta observe, à la dissection de deux Hirudo officinalis, 7o jours après les avoir infestées sur une grenouille parasitée, deux larves de nématodes dont la longueur est environ le double de celle des microfilaires : il conclut alors à la possibilité de l'évolution de la filaire chez les Hirudinées et considère, en particulier, Haemopis sanguisuga comme pouvant en etre le vecteur normal.

(a) Il semble, par contre, qu'aucune expérience n'ait été tentée sur ce point avec des Phlébotomes. 
compte; néanmoins, nous noterons que jamais au cours des heures passées auprès de grenouilles fixées au bord des mares, souvent dans les clairières des bois, nous n'avons observé se posant sur elles un seul Culicidé (ces observations étant effectuées le jour seulement, il est bien évident que nous ne parlons ici que d'espèces diurnes). Par contre, nous avons récolté, dans ces conditions, d'une part, en très grand nombre, Forcipomyia velox Winn. 1852, ainsi que nous l'avons indiqué en r 940 (C. Desportes et H. Harant), d'autre part, de petits brachycères, que provisoirement nous rapportons au genre Atrichops Verrall. et découverts en juillet à Morlanne (région de Pau). Disons immédiatement que les expériences de transmission d'Icosiella neglecta par ces Rhagionidx (= Leptididx auct.) sont restées négatives. Nous avons en effet pu capturer une trentaine de ces mouches - toutes femelles - alors qu'elles se gorgeaient avidemment sur les grenouilles parasitées (I) : la dissection de la moitié d'entre elles, tuées les jours suivants, a montré un grand nombre de microfilaires ingérées qui succombaient toutes, sans évoluer, vers le cinquième jour.

Les premières recherches sur Forcipomyia velox, interrompues en septembre 1939 , nous ayant appris que les embryons d'Icosiella neglecta vivaient parfaitement et subissaient un début d'évolution dans le corps de ces moucherons, 53 heures après l'ingestion, nous avons repris ces recherches pendant l'été I 940.

Les méthodes utilisées pour l'infestation et la capture des Forcipomyies furent les mêmes que celles exposées dans la note de 1940 et qui nous avaient permis d'étudier la biologie de ces nématocères : elles présentent l'avantage de placer l'expérimentation dans des conditions quasi-naturelles, puisque le lien retenant les grenouilles infestées au bord des mares ne peut en aucune manière apporter de modifications à ces conditions. La photographie de la page 62 , que nous devons à l'amabilité de notre ami R. Laurent, permet de se rendre compte de la façon dont s'opère cette infestation des moucherons venus spontanément prendre leur repas sur l'hôte. Lorsque ce repas, opéré simultanément par plusieurs animaux semble toucher à sa fin, ils sont capturés au moyen de l'entonnoir retourné.

Si de cette manière, il nous a été possible d'obtenir un grand nombre de Forcipomyia, parfois plus d'une centaine en quelques heures (2),

(1) Ces diptères ne s'attaquaient pas à l'homme.

(2) Lorsque le temps est particulièrement ensoleillé et que les femelles viennent en grand nombre piquer les grenouilles, il est également possible de récolter quelques mâles sur les ombellifères qui croissent au voisinage de la mare : c'est ainsi que nous avons pu obtenir deux individus de ce sexe sur les fleurs de la Grande Berce (Heracleum sphondylium L.) (fig. 3-A). 
jamais, par contre, nous n'avons pu les conserver jusqu'à complète évolution des larves d'Icosielles et quel que fùt le modèle de cage réalisé au cours de cette première série d'expériences, les mouches succombaient entre le troisième et le cinquième jour, c'est-à-dire dans un laps de temps au bout duquel les larves de filaires sont loin d'être infectieuses. Ainsi, les mèmes difficultés auxquelles se heurtait D. B. Blacklock en cherchant à réaliser le cycle expérimental d'Onchocerca volvulus chez Simulium damnosum (premiers travaux) se présentaient dans le cas d'Icosiella neglecta. Néanmoins, nous pouvons donner, sur l'évolution de ce nématode, quelques précisions résultant des observations faites au cours de septembre r 940 . Nous exprimons notre vive reconnaissance au professeur E. Brumpt, pour l'intérêt qu'il a bien voulu porter au présent travail, en mettant à notre disposition tout le matériel et les ouvrages qui nous étaient nécessaires, tant à la Station de Richelieu qu'à l'Institut de Parasitologie de Paris.

1. Examen de Forcipomyia isolèes après les repas infectieux. - La quantité de larves ingérées au cours d'un repas, pris sur des grenouilles généralement bien parasitées, n'est jamais très élevée (I) : nous n'en avons jamais rencontré plus de cinq ou six par exemplaire; beaucoup de moucherons n'étaient pas parasités (les animaux capturés par lots de ro à 20 , ou davantage, étant très différemment gorgés, il est à peu près impossible de donner actuellement un pourcentage d'infestation consécutive à ces repas).

Les larves puisées au cours d'un mème repas subissent toutes très rapidement - déjà dans le sang en voie de digestion - un commencement d'évolution se traduisant par des modifications plus ou moins considérables selon les individus. Peut-être même, cette évolution cesse-t-elle complètement pour certaines larves et que toutes ne parviennent pas au stade infectieux? Il est difficile de le dire dans de telles conditions d'observation, cependant jamais nous n'avons rencontré de larves mortes dans le corps de Forcipomyies sacrifiées.

La première modification subie par la larve est un raccourcissement et un épaississement de tout le corps, sauf de la partie postérieure : celle-ci est aciculée alors que l'extrémité antérieure est bien

(I) Nous nous efforcerons d'établir, au cours de la campagne de rgár, le rapport entre la quantité de larves ingérées par les moucherons et le nombre de microfilaires chez l'hòte, afin de voir s'il y a, comme dans le cas de Wuchereria bancrofti (Cobbold), Onchocerca volvulus Leuckart ou Onchocerca caecutiens Brumpt, concen. tration des embryons au point de piqùre. 


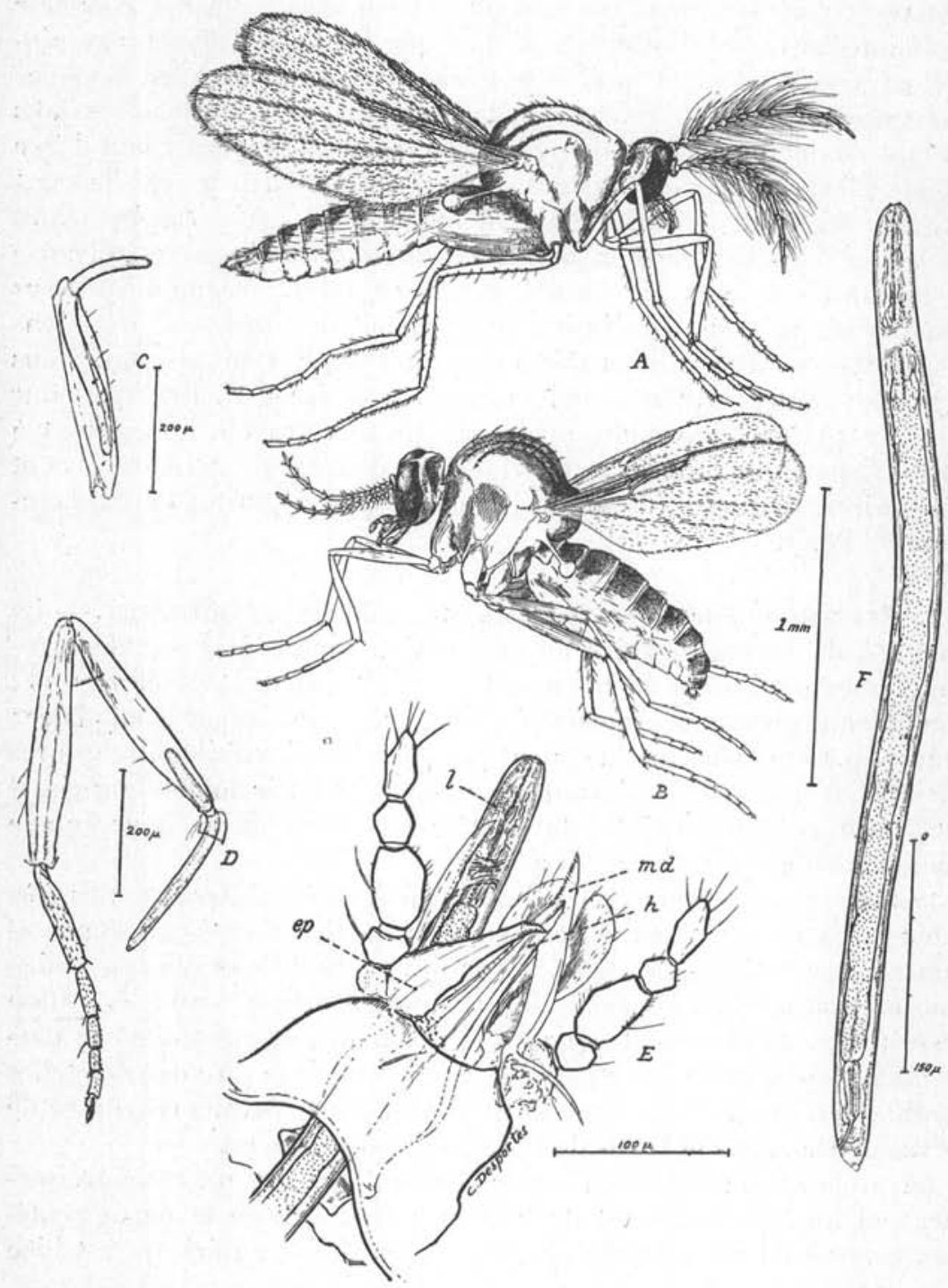

Fic. 3. - A, Forcipomyia velox Winn. $1852 \sigma^{7}$; B, Forcipomyia velox Winn. $185_{2}$ \& ; C-D, grandes larves de provenance spontanée, logées dans les pattes de Forcipomyia velox; E, larve infectieuse $(l)$ émergeant des pièces buccales de Forcipomyia velox ( $m d$, mandibules; $e p$, épipharynx; $h$, hypopharynx); F, larve infectieuse. 
arrondie : l'accroissement de diamètre ne s'accompagne, à ce stade, d'aucune modification appréciable de l'organisation interne.

Les chiffres suivants donnent une idée des modifications de taille que subissent les larves dans le corps de leur hòte pendant les premières heures (fig. 4 B.F).

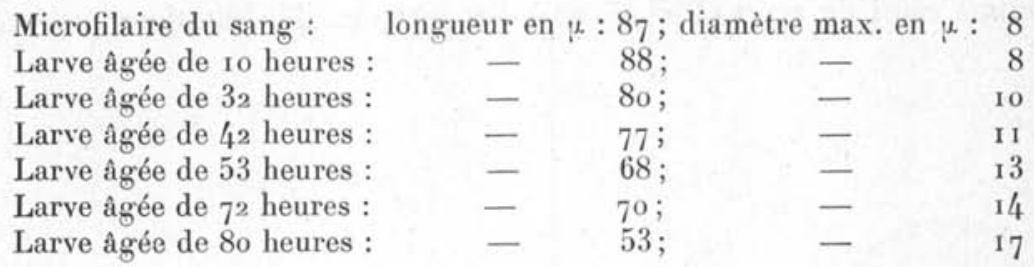

Il semble donc que, très tôt, les larves tendent vers la forme dite en saucisse : les mouvements dont sont douées ces jeunes formes sont moins vif́s que ceux des microfilaires du sang et se traduisent en général par des déplacements latéraux des extrémités, plus particulièrement de l'extrémité antérieure.

2. Examen de Forcipomyia spontanèment infestées. - Les dissections d'animaux spontanément infestés, entreprises en septembre r 940 seulement, permirent de découvrir des larves de nématodes généralement très évoluées, parvenues la plupart au stade infectieux et localisées soit dans les muscles du thorax ou des pattes (fig. $3 \mathrm{C}-\mathrm{D}$ ), soit dans la tête : nous avons mème observé dans un cas une larve infectieuse engagée entre les pièces buccales (fig. $3 \mathrm{E}$ ) : il est certain que la sortie de la larve, dans le cas particulier, a été déterminée par la pression de la lamelle, mais ce mème processus doit vraisemblablement se produire au moment de la piqùre de l'hòte par l'insecte : nous ne pouvons autrement expliquer que par cette effraction la sortie de larves dont le diamètre est supérieur à celui des palpes et des différentes pièces buccales de l'hôte intermédiaire.

Les plus grandes de ces larves atteignent $583 \mu$ avec un diamètre maximum de $33 \mu$ (fig. $3 \mathrm{~F}$ ) ; leur tube digestif, pourvu d'un anus, et l'anneau nerveux sont bien apparents ; les ornementations cuticulaires sont représentées par une fine striation transversale, deux dents antérieures, l'une dorsale, l'autre ventrale (fig. 2 G) et deux mamelons caudaux latéraux (fig. : $\mathrm{H}$ ).

Les formes les moins évoluées, encore au stade dit "en saucisse " mesurent $3{ }_{2} 5 \times 35 \mu$; l'ornementation cuticulaire de ces formes peu mobiles est très peu différenciée (fig. $4 \mathrm{~A}$ ). 
Comme l'on peut s'en rendre compte, il existe entre ces larves de provenance spontanée et les larves obtenues par repas infectieux contrôlés, des différences très marquées : aucun terme de transition n'a pu être mis en évidence. Avec toute la réserve que comporte l'interprétation de telles constatations, dues pour une grande part au trop petit nombre de dissections, nous rappellerons qu'au cours d'un été nuageux comme celui de 1940 (Richelieu) à des périodes d'infestations plus ou
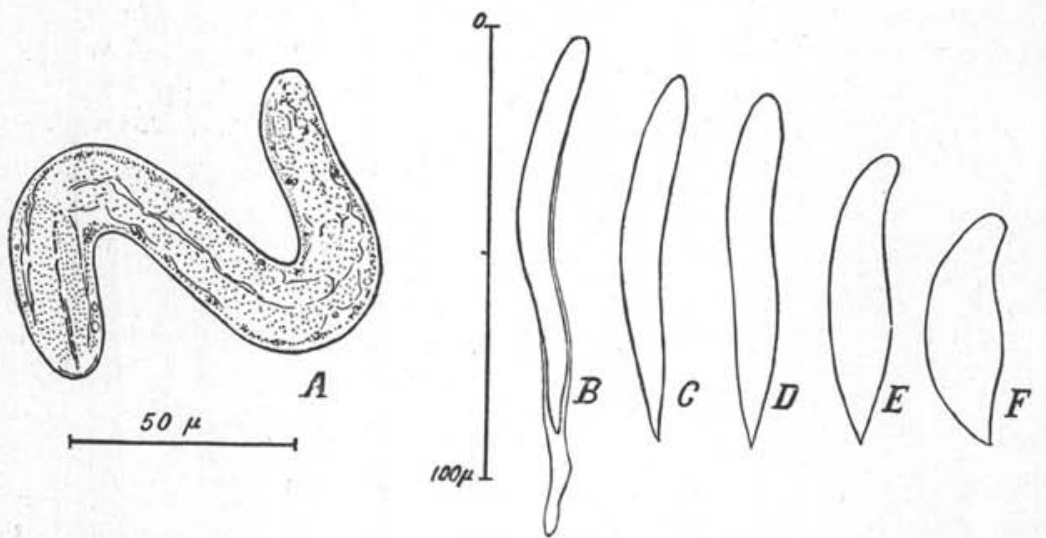

Fig. 4. - A, larve au stade dit "en saucisse ", trouvée dans le corps de Forcipomyia velox spontanément infestée; B-F, figures schématiques représentant une microfilaire (B) d'Icosiella neglecta et les modifications qu'elle subit dans le corps de Forcipomyia velox 32 heures (C), 42 heures (D), 72 heures (E) et 80 heures (F) après son ingestion (expérimentale).

moins générales peuvent parfaitement succéder des périodes négatives : nous avons en effet déjà signalé l'action exercée par les conditions atmosphériques sur la biologie de Forcipomyit. L'absence de larves à certains stades peut également être liée dans une certaine mesure à un caractère de périodicité saisonnière des filaires chez les grenouilles, périodicité déjà notée dans le Catalogue des Entozoaires de Vienne, puis par de Nabias et Sabrazès (in R. Blanchard, I 895 ), également remarquée depuis trois années pour les microfilaires par nous-mêmes.

3. Pourcentage et taux d'infestation spontanée de Forcipomyia par des larves de Nématodes. - A la dissection d'un lot de I3o Forcipomyia, capturées sur des grenouilles vertes infestées ainsi que nous l'avons indiqué et disséquées dans les premières heures suivant le repas, 17 fois, nous avons rencontré des larves : ro de ces cas étaient représentés par les jeunes formes d'Icosiella ingérées au 
moment de la capture, les 7 autres par les larves de nématodes infectieuses, soit seules, soit en association avec les précédentes. Ainsi, consécutivement aux infestations spontanées, contractées au cours de repas vraisemblablement complets, 5,3 o/o des Forcipomyia hébergent des formes évoluées; une même femelle renferme tantôt une seule de ces larves ( 3 cas), tantôt deux ( 2 cas), trois ( 1 cas) ou quatre ( $\mathrm{r}$ cas).

Le pourcentage d'infestation spontanée est peut-être plus élevé : il faut en effet tenir compte de la pénétration possible de larves - si elles sont bien celles d'Icosiella - chez les grenouilles pendant le laps de temps qui précède la capture (cette pénétration chez des animaux déjà parasités doit ètre possible : des surinfections expérimentales au moyen d'adultes ont été réalisées par F. Coutelen (r928); en outre, le nombre de filaires est toujours plus élevé chez les grosses grenouilles).

4. Tentative de pénétration chez les grenouilles des larves infectieuses. - L'identité morphologique entre les larves infectieuses spontanées et les microfilaires d'Icosiella ingérées n'ayant pu être établie par suite du manque de matériel, nous avons essayé de faire évoluer ces larves vers une forme reconnaissable.

Le seul spécimen qui nous restait alors était une larve considérée comme parfaitement infectieuse et bien mobile; prélevée dans une goutte d'eau physiologique, elle fut placée sur la queue transparente d'une jeune grenouille verte. Mue par un tropisme évident, la larve, devenue alors très active, frappa inlassablement de la tête les téguments lisses de l'hòte présenté pendant 20 minutes, cherchant soit linterstice favorable à une pénétration, soit un point d'appui permettant une perforation active. Ces conditions, fournies par l'hòte intermédiaire, n'étant pas réalisées expérimentalement, nous avons voulu mécaniquement les produire : c'est au cours de cette dernière manipulation que la larve fut perdue.

\section{Superposition entre la répartition géographique de Forcipomyia} et d'Icosiella. - Les seules constatations que nous ayons pu faire à ce sujet et qu'il aurait été intéressant d'amplifier, sont les suivantes :

A. - Basses-Pyrénées (Morlanne, juillet 1940).

Forcipomyia velox est très abondant;

1 I grenouilles sur 19 renferment des filaires;

4 grenouilles sur 6 renferment des filaires;

4 grenouilles sur 4 renferment des filaires. 
B. - Charente-Inférieure (énvirons de Fouras, août r940). Forcipomyia velox n'a pas été rencontré;

8 grenouilles $\operatorname{sur} 8$ sont négatives.

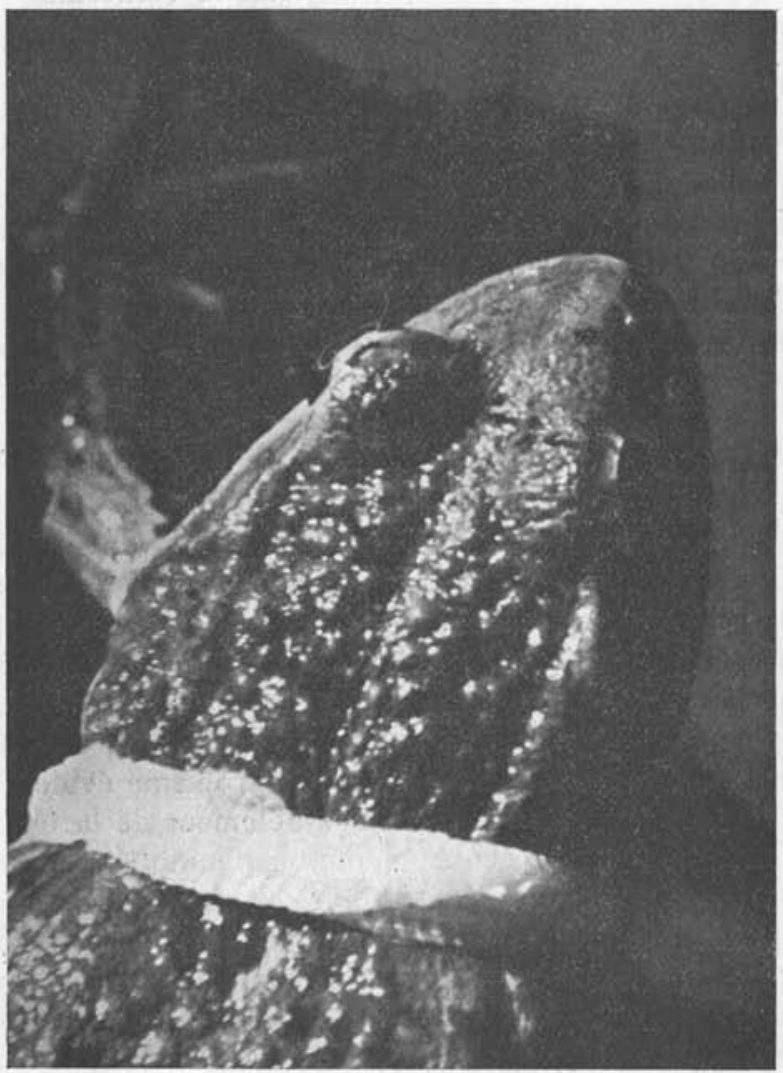

Fig. 5. - Forcipomyia veloæ venues spontanément piquer une grenouille verte fixée (Photographie communiquée par $\mathbf{R}$. Laurent).

C. - Indre-et-Loire (Richelieu et environs, septembre 1940 ). Forcipomyia velox est abondant;

$33 \mathrm{o} / \mathrm{o}$ des grenouilles renferment des filaires.

Conclusions. - Avec toutes les réserves qu'il convient de faire en présence de telles constatations, nous considérons actuellement For- 
cipomyia velox Winn. I852 comme étant le vecteur normal d'Icosiella neglecta (Diesing $\mathrm{I} 85 \mathrm{r}$ ), filaire commune du tissu souscutané et conjonctif intramusculaire de Rana esculenta L., parce que :

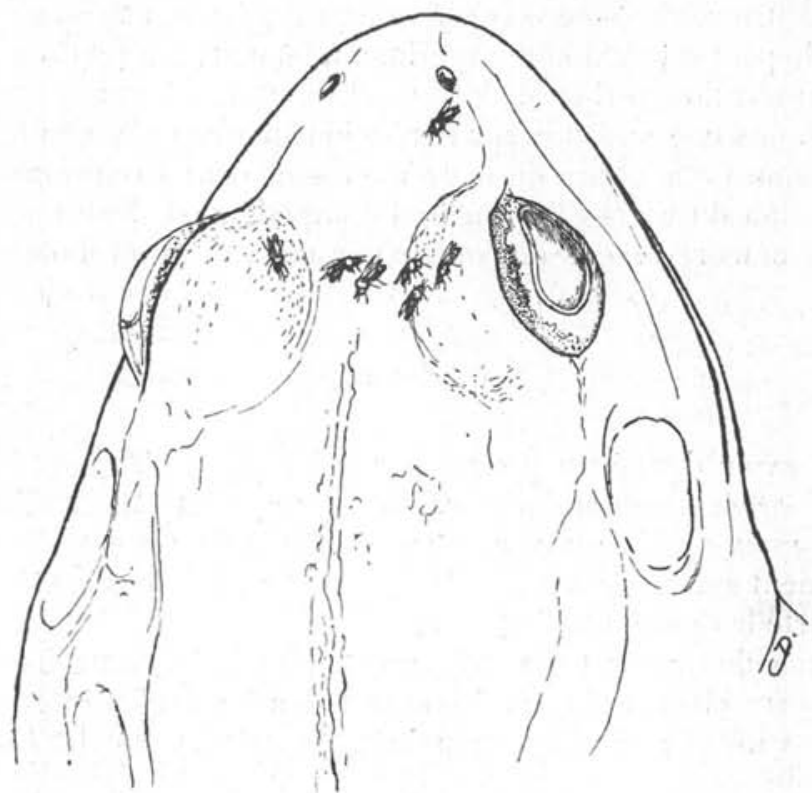

Fıg. 6. - Figure schématique indiquant la position des Forcipomyia sur la tête de la grenouille photographiée.

$\mathrm{I}^{0}$ Forcipomyia velox ne semble se nourrir que sur les batraciens;

$2^{\circ}$ Forcipomyia velox ne s'éloigne pas du voisinage des mares (ces deux points ont été établis antérieurement, cf. C. Desportes et H. Harant 1939 );

$3^{\circ}$ Le seul batracien susceptible d'être piqué dans ces conditions, par Forcipomyia velox est Rana esculenta;

$4^{\circ}$ Les autres diptères hématophages, suçant le sang des grenouilles sont beaucoup plus rares que Forcipomyia velox et malgré différentes tentatives, nous n'avons jamais obtenu, jusqu'à présent, qu'une petite mouche (Atrichops sp.), chez qui l'évolution ne peut se faire, les microfilaires ingérées succombant le cinquième jour ;

$5^{\circ}$ Les microfilaires d'Icosiella neglecta, ingérées par Forcipomyia velox, au lieu de succomber, commencent rapidement leur évolution; 
$6^{\circ}$ Spontanément, plus de 5 o/o de femelles de Forcipomyia velox hébergent des larves de nématodes sur lesquelles Rana esculenta exerce un tropisme positif évident;

$7^{\circ}$ Rana temporaria, que le mode d'existence n'expose en aucune saison aux piqûres de Forcipomyia velox, n'héberge jamais spontanément, à notre connaissance (I), Icosiella neglecta, alors que la même grenouille peut servir d'hôte expérimental à cette filaire (Coutelen 1928 et communication verbale).

Néanmoins une certitude sur le rôle joué par ce Chironomide dans la transmission de la filaire de la grenouille ne peut être acquise que par la réalisation du cycle expérimental complet, c'est donc dans ce sens que nous nous réservons de donner une suite à ces premières recherches.

\section{RÉSumÉ}

Après avoir donné un aperçu sur l'historique d'Icosiella neglecla (Diesing) souvent confondue avec Filaria rubella Rud., nous apportons quelques précisions sur la morphologie de cette filaire, et plus spécialement sur les caractères de l'armature génitale du mâle : celleci comporte les particularités suivantes :

$\mathrm{I}^{\circ}$ Le spicule droit est généralement le plus long, contrairement à ce qui s'observe chez la plupart des nématodes à spicules inégaux.

$2^{\circ}$ Dans un cas sur sept examinés, le spicule gauche est le plus long.

$3^{\circ}$ L'inversion dans la disposition des spicules est accompagnée d'une inversion dans la forme asymétrique du petit spicule.

$4^{\circ}$ Le grand spicule, en s'évaginant, s'engage dans la gouttière du petit spicule qui, le retenant par deux petits becs, semble lui servir de guide en l'absence de gorgeret.

Des recherches sur l'évolution d'Icosiella neglecta nous amènent à considérer - avec toutes les réserves qu'il convient de faire en présence de telles recherches - qu'elle admet pour vecteur normal Forcipomyia velox Winn. 1852, Chironomide Cératopogoniné dont les femelles hématophages semblent ne piquer exclusivement que les batraciens situés au bord des mares et sucent normalement le sang de Rana esculenta L. : les microfilaires ingérées par ces moucherons demeurent parfaitement vivantes et commencent rapidement leur évo-

(1) De Nabias et Sabrazès signalent cependant l'existence de filaires chez les « quelques grenouilles rousses (Rana temporaria) qui avaient été prises dans le mème étang que les précédentes (Rana esculenta) ». 
lution, tendant vers la forme dite " en saucisse ". Les formes évoluées ne peuvent être obtenues par suite de la mort vers le cinquième jour des moucherons en captivité, mais 5,3 o/o des femelles, capturées dans la nature, hébergent des larves infectieuses de nématodes, au nombre de I à 4 par hôte : une de ces larves, placée sur la queue d'une jeune Rana esculenta cherche visiblement à pénétrer chez l'hôte présenté, malgré les conditions peu favorables dans lesquelles elle se trouve. Enfin une certaine corrélation semble exister entre la répartition géographique du parasite et celle de ce moucheron.

\section{BibliographIE}

BLACKLock (D. B.). - The development of Onchocerca volvulus Leuckart in Simulium damnosum. Ann. Trop. Med. and Parasit., XX, r926, p. I.

Blanchard (R.). - Les Vers du Sang. Les Hématozoaires de l'Homme et des Animaux, Paris, 1895 , p. 181 .

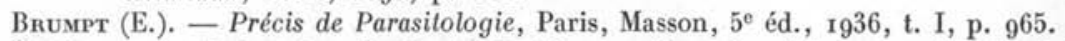

Catalogue des Entozoaires du Musée de Vienne, 1811, in : Westrumr (A. H. L.). - De Helminthibus acanthocephalis, $182 \mathrm{I}$, p. 78 .

Causey (O. R.). - Aëdes and Culex mosquitoes as intermediate hosts of frog filaria, Foleyella sp. Amer. Journ. Hyg., XXIX-C, 1939, p. 79.

- Development of the larval stages of Foleyella brachyoptera in mosquitoes. Amer. Journ. Hyg., XXX-D, r939, p. 69.

Chrtwoon (B. G.) et CHrwwoon (M. B.). - An Introduction to Nematology, I, 2, I 938 , p. 120.

Coutelen (F.). - Contribution à l'étude de la filariose des grenouilles. Infection et surinfection expérimentales. Ann. de Parasit., VI, 1928, p. 196.

Dr Nabins et Sabrazìs. - La filaire du sang des grenouilles. Découverte du mâle. Assoc. franç. pour l'Avancement des Sciences, Congrès de Pau, 1892, p. $\mathbf{I}$.

Despontes (C.) et Harant (H.). - Observations sur la biologie d'un Ceratopogoniné hématophage, Forcipomyia velox Winn. $185_{2}$, piqueur de la grenouille verte. Ann. de Parasit., XVII, 1939-I940, p. 369 .

Dirsing (K. M.). - Systema Helminthum, Vienne, 1851, t. II, pp. 269, 276, 284.

Dujardis (F.). - Histoire naturelle des Helminthes, 1845, p. 59.

Linstow (O. von). - Parasitische Nematoden. Die Süszwasserfauna Deulschlands,

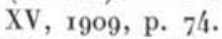

Matmos (C.) et LÉGER (M.). - Recherches de Parasitologie et de Pathologie humaines et animales au Tonkin, Paris, Masson, 1911, pp. 406, 450 .

Porтs (A.). - Ricerche sul ciclo exolutiva della Filaria rubella Rud. Zool. Anzeig., XXXIX, 1912, p. 202.

Ralleiet (A.). - Sur les Filaires de Batraciens. Bull. Soc. Path. Exol., IX, 1916, p. 137 .

Rudolphi (C. A.). - Entozoorum Synopsis, 1819, pp. 5, 1о, 212.

Seurat (L. G.). - Sur deux Filaires des reptiles du Nord-Africain. C. R. Soc. Biol., LXXIX, 1916, p. 1131.

ANxales de Pakasttologie, T. XVIII, N ${ }^{\circ 3}$ 1-2-3. $-194 \mathrm{t}$. 
- Filaires des Reptiles et des Batraciens. Bull. Soc. Hist. Nat. Afr. Nord, VIII, $19 \times 7$, p. $2 x$.

Sknjabin (K. I.) et Scriknobalova (N. P.). - Contribution au remaniement de la classification des nématodes de l'ordre des Filariata Skrjabin r9r5. Ann. de Parasit., XIV, I936, p. 6r.

VALEntın (G.). - Erzeugung der Eingeweidewürmer. Repertorium für Anatomie und Physiologie, VII, 184r, p. $5 \mathrm{r}$.

Vogr (C.), - Beitrag zur Entwicklungsgeschichte der Filarien. Archiv f. Anat. Phy. siol. u. Wiss. Med, J. Müller, 1842, p. 189 .

Vulpian (E. F.). - Note sur les hématozoaires filiformes de la grenouille eommune. C. R. Soc. Biol., 1854, p. 123.

Walton (A. C.). - The Nematoda as Parasites of Amphibia. Journ. Parasit., XXI, 1935, p. 27 .

- A Revision of the Nematodes of the Leidy Collections. Acad. Nat. Sci. Phila., LXXIX, r927, p. III.

WEHR (E. E.). - A revised classification of the nematode superfamily Filarioidea. Proc. Helm. Soc. Wash., II, 1935, p. 84.

Yorke (W.) et Maplestone (P. A.), - The nematode Parasites of Vertebrates, Londres, 1926, pp. 3955,411 , 424.

Institut de Parasitologie de la Faculté de médecine de Paris et Station expérimentale de Richelieu (Indre-et-Loire)

(Directeur : Professeur E. Brumpt). 